\title{
Performance Analysis Of Channel Capacity AND THROUgHPUT OF LTE DOWNLINK SYSTEM
}

\author{
P.Poornima ${ }^{1}$, G. Laxminarayana ${ }^{2}$ and D. Srinivas Rao ${ }^{3}$ \\ ${ }^{1}$ Associate Professor, Dept. of ECE, Sphoorthy Engineering College, Hyderabad, India \\ ${ }^{2}$ Professor, Dept. of ECE, Anurag College of Engineering, Hyderabad, India \\ ${ }^{3}$ Professor, Dept. of ECE, JNTUH CEH, Kukatpally, Hyderabad, India
}

\begin{abstract}
In this paper, we analyzed a numerical evaluation of the performance of MIMO radio systems in the LTE network environment. Downlink physical layer of the OFDM-MIMO based radio interface is considered for system model and a theoretical analysis of the bit error rate of the two space-time codes $($ SFBC $2 \times 1$ and FSTD $4 \times 2$ codes are adopted by the LTE norm as a function of the signal to noise ratio. Analytical expressions are given for transmission over a Rayleigh channel without spatial correlation which is then compared with Monte-Carlo simulations. Further evaluated channel capacity and simulation results show throughput almost reaches to the capacity limit.
\end{abstract}

\section{KEYWORDS}

Channel Capacity, MIMO, OFDM, LTE Downlink, Spate time Block Coding and Throughput.

\section{INTRODUCTION}

LTE is designed to meet carrier needs for high-speed data and media transport as well as highcapacity voice support well into the next decade. The LTE PHY employed some advanced technologies like Orthogonal Frequency Division Multiplexing (OFDM) and Multiple Input Multiple Output (MIMO) to provide downlink speed up to $100 \mathrm{Mbps}$ [1-3]. The combination of OFDM and MIMO with spatial multiplexing (SM) scheme is viewed as a viable means to achieve high user information rates.

In cellular networks, the same radio spectrum is reused in different cells, in order to improve the network capacity [5,6]]. Several authors have considered the effect of increasing the density of base stations in the network, and improvements in the network capacity with increasing base station density have been revealed [7]. For a MIMO LTE system, a significant spectral efficiency improvement of $17 \%$ over SIMO LTE for static scenarios is achieved though slightly over $29 \%$ performance drop occurs when a mobility of $29 \mathrm{mph}$ is introduced $[9,10,11]$. Hence it is established that in deploying diversity techniques, spectral efficiency is not the target rather diversity gain is. In $[11,12]$, a performance of $2 \times 2$ STBC and SM are evaluated using typical OFDM based system Receiver Transmitter Channel Coding Modulation Mapper S/P Converter IFFT P/S Converter Channel Decoding Modulation Damper P/S Converter IFFT S/P Converter Wireless Channel with AWGN throughput and BER as metrics of measurement. In $[13,14,15]$ the performance of MIMO in LTE is viewed from the capacity perspective.

DOI: $10.5121 /$ ijcnc.2017.9505 
Despite of these advantages due to the combination of OFDM and MIMO in the physical layer, there are many problems that are to be answered to achieve data rate as per the standards. The established distribution of resources allows to utilize system capacity for the evaluation of performance $[2,3]$. This paper analyses channel capacity for the MIMO-OFDM system using with spatial multiplexing and

This paper is organized as In section II, channel capacity analysis of LTE system with spatial multiplexing and diversity techniques. Section III, A framework on channel capacity with space time coding scheme with SFBC and FSTD is presented. Throughput analysis for the various MQM schemes under proposed space time coding is represented in Section IV .In Section V, we present numerical analysis and simulation results depicting the performance of the proposed system. Section VI, we conclude.

\section{Channel Capacity Analysis For Lte Systems}

The channel capacity of SISO systems is described by the information theory based on the mathematical frame work introduced by Shannon [5]. In fact, it is the theory of information that communication systems have evolved to their present form. MIMO systems are no exception to this rule since Telstar $[6,7]$ has extended the work of Shannon to the case of multiple antennas and on OFDM case. As an application of the theory, we will consider first the channel capacity for the spatial multiplexing MIMO scheme followed by the channel capacity of the different diversity schemes.

\section{A. Channel Model And Channel Capacity Of Spatial Multiplexing Scheme}

MIMO channel capacity for single carrier system and in Rayleigh channel considered in multi antenna systems. Where let us consider vector transmission model with $N_{t} \mathrm{t}$ transmits antennas and $N_{r}$ receive antennas, then the received signal is expressed as

$$
y=\sqrt{\frac{\rho}{N_{t}}} H+n
$$

In the above model, $\rho$ is the average signal to noise ratio and $y$ represents the received vector of size $N_{r} \times 1$ and $s$ represents the transmitted vector of size $N_{t} \times 1$. The MIMO channel is represented by the $H$ matrix of size $N_{r} \times N_{t}$, whereas the noise is represented by the vector $\mathrm{n}$ of size $N_{r} \times 1$. The mutual information between the transmitted signal and the received signal, I $(s ; y)$ when the channel matrix $H$ is deterministic and is known to the receiver which is given as:

$$
I(s ; y)=\log _{2}\left[\operatorname{det}\left(I_{N_{r}}+\frac{\rho}{N_{t}} H C_{s} H^{H}\right)\right] \quad(\text { bps } / H z)
$$

Where Cs is the covariance matrix of transmitted signal vector $s$ and $I$ is the identity matrix with dimension $N_{r} . \rho$ is the average signal to noise ratio. By definition, the channel capacity is the maximum of the mutual information where the maximization is taken over all possible covariance matrixes $C_{s}$ and hence the deterministic MIMO channel capacity can be written as:

As is the covariance matrix of transmitted signal vector $s$ and $I$ is the identity matrix with dimension $N_{r} . \rho$ is the average signal to noise ratio. By definition, the channel capacity is the 
maximum of the mutual information where the maximization is taken over all possible covariance matrix $C_{s}$ and hence the deterministic MIMO channel capacity can be written as:

$$
\begin{gathered}
C(H)=\max _{p(s)} I(s ; y) \quad(\text { bps / Hz }) \\
C(H)=\max _{t_{r} C_{S}=N_{t}} \log _{2}\left[\operatorname{det}\left(\frac{I}{N_{r}}+\frac{\rho}{N_{t}} H C_{S} H^{H}\right)\right] \quad(b p s / H z)
\end{gathered}
$$

For fading channel, the channel matrix $H$ is a random matrix and hence the associated channel capacity is also a random variable. To deal with the random behavior of the channel of the channel, the average of the above equation over the distribution of $H$ with the given name of erotic MIMO channel capacity can be defined as:

$$
C_{E}=E\left\{\max _{t_{r} C_{S}=N_{t}} \log _{2}\left[\operatorname{det}\left(\frac{I}{N_{r}}+\frac{\rho}{N_{t}} H C_{S} H^{H}\right)\right]\right\} \quad(b p s / H z)
$$

$\mathrm{T}$ the above derivation of the erotic MIMO channel capacity does not provide information to choose the covariance matrix of $s\left(C_{s}\right)$ to get the maximum mutual information. To be able to compute the maximization, it should be clarified if the transmitter, the receiver, or both have perfect knowledge of the channel state information (CSI).If the channel matrix $H$ is known at the transmitter, the transmit covariance matrix $\left(C_{s}\right)$ can be chosen to maximize the channel capacity for a given realization of the channel. If the channel matrix $H$ is, however, known at the receiver, the optimal signal covariance matrix has to be chosen according to:

$$
C_{S}=I
$$

With such covariance matrix, the erotic MIMO channel capacity becomes]:

$$
C_{E}=E\left\{\log _{2}\left[\operatorname{det}\left(\frac{I}{N_{r}}+\frac{\rho}{N_{t}} H C_{s} H^{H}\right)\right]\right\} \quad(\text { bps / Hz })
$$

\section{B. Channel Model And Channel Capacity Of Diversity Schemes}

The erotic channel capacity in (7) is valid for channel matrix where different signals are transmitted independently and hence cannot be applied directly to the space time block coding where the signals are transmitted form of spatial block codes. In fact, an STBC scheme with Nt transmit with $N_{t}$ transmit antennas and $N_{r}$ receive antennas is generally characterized by the transmission matrix which has the general form $[9,10]$ :

$$
G=\left(\begin{array}{cccc}
g_{11} & g_{21} & \ldots & g_{N_{t 1}} \\
g_{12} & g_{22} & \ldots & g_{N t 2} \\
\cdot & \cdot & \ldots & \cdot \\
g_{1 T} & g_{2 T} & \ldots & g_{N_{t} T}
\end{array}\right)
$$


Where gig represents a linear combination of the signal constellation components and their from conjugates. The gig is the $i^{\text {th }}$ transmit antenna in the $j^{\text {th }}$ time slot for $\mathrm{i}=1, \ldots, \mathrm{Nt}$ represents the number of time slots used to transmit $S$ symbols. For such STBC, the equivalent AWGN scaled channel is given by:

$$
y_{n T}=\frac{1 \ln }{R_{C}}\|H\|_{F}^{2} x_{n T}+w_{n T}
$$

Where, and is the $S \times 1$ complex matrix after STBC decoding and $S$ represents the number of transmitted symbols. And is the transmitted $S \times 1$ complex vector with each entry having energy $E_{s} / N_{\mathrm{t}}, E_{s}$ is the maximum total transmitted energy on the $N_{t}$ transmit antennas per symbol time, and $w_{n T}$ is complex Gaussian noise with zero mean and variance $N_{0} / 2$ in each dimension. $\|H\|_{F}^{2}=\sum_{i=1}^{N} \sum_{j=1}^{M}\left\|h_{i j}\right\|^{2}$ is the squared Fresenius norm of $H, h_{i j}$ is the channel gain from the $i^{t h}$ transmit antenna to the $j^{\text {th }}$ receive antenna. $R_{c}$ is the code rate of the STBC and defined as $S / T$, where $T$ is the number of time slots to transmit one block code. From the equivalent AWGN channel in (9), it is shown that the effective instantaneous SNR at the receiver denoted as $\gamma$ is given as:

$$
\gamma=\frac{E_{S}}{N_{t} R_{C} N_{O}}\|H\|_{F}^{2}
$$

which means that the channel matrix is converted into a scalar channel and hence the ergodic capacity of the equivalent STBC channel in (9) is given by:

$$
\bar{C}=E\left[R_{c} \log _{2}(1+\gamma)\right] \quad(\text { bps } / H z)
$$

or equivalently, by inserting (10) into (11):

$$
\bar{C}=E\left[R_{C} \log _{2}\left(1+\frac{E_{S}}{N_{t} R_{C} N_{O}}\|H\|_{F}^{2}\right)\right] \quad(\text { bps / Hz })
$$

If the probability density function of the instantaneous $\operatorname{SNR} p\left(\gamma_{s}\right)$ is known, the ergodic capacity of the equivalent STBC can be evaluated using the following integral equation for capacity evaluation:

$$
\bar{C}=R_{c} \int_{0}^{\infty} \log _{2}(1+\gamma) p\left(\gamma_{S}\right) d \gamma_{S} \quad \text { (bps / Hz) }
$$

\section{Channel Capacity analysis For Space Time Codes:}

\section{A. Sfbc Channel Capacity analysis}

Since OFDM system converts the multipath channel into $N$ frequency flat-fading sub- channels, the average capacity over flat-fading sub-channel that is defined by $C_{k}$ is determined, then, the overall average channel capacity over $N$ can be found as: 


$$
\bar{C}^{\chi}=\frac{1}{N} \sum_{k=1}^{N} \bar{C}_{k}^{\chi}
$$

Where, $k$ represents the subcarrier index and $\chi \in\{$ SFBC, FSTD $\}$. The average capacity achieved by a SFBC-OFDM scheme is calculated using the probability density function of the instantaneous SNR:

$$
\bar{C}_{k}^{S F B C}=\int_{0}^{\infty} R_{c} \log _{2}(1+\gamma) f_{\gamma}^{S F B C}(\gamma) d \gamma
$$

Where $f_{\gamma}^{S F B C}(\gamma)$ is defined as

$$
f_{\gamma}^{S F B C}(\gamma)=\frac{2}{\gamma^{2}} \gamma^{e^{-\frac{2}{\gamma} \gamma}}
$$

Substituting the PDF in the equation of average capacity, we obtain:

$$
\bar{C}_{k}^{S F B C}=\int_{0}^{\infty} R_{C} \log _{2}(1+\gamma) \frac{2}{\bar{\gamma}^{2}} \gamma^{e^{-\frac{2}{\gamma} \gamma}} d \gamma
$$

The equation of the $\bar{C}_{k}^{S F B C}$ can be written as:

$$
\bar{C}_{k}^{S F B C}=A \mu \int_{0}^{\infty} \ln (1+\gamma)(\mu \gamma)^{\eta-1} e^{-\mu \gamma} d \gamma
$$

Where

$$
A=\frac{R_{C} \times \log _{2}(10) \times \log _{10}(e)}{2} ; \mu=\frac{2}{\gamma} ; \eta=2
$$

The integral in equation (18) can be evaluated using the following:

$$
\frac{\mu}{(\eta-1) !} \int_{0}^{\infty} \ln (1+x)(\mu x)^{\eta-1} e^{-\mu x} d x=p_{\eta}(-\mu) E_{1}(\mu)+\sum_{q=1}^{\eta-1} \frac{1}{q} p_{q}(\mu) p_{\eta-q}(-\mu)
$$

Where, $p_{q}($.$) and E_{1}(x)$ represent respectively the Poisson distribution and the exponential of the first order which is given as:

$$
\begin{gathered}
p_{q}(x)=\sum_{v=0}^{q-1}\left(\frac{x^{v}}{v !}\right) e^{-x} \\
E_{1}(x)=\int_{x}^{\infty} t^{-1} e^{-t} d t \quad \text { for } x>0
\end{gathered}
$$


Using (20), the closed form of (18) becomes

$$
\bar{C}_{k}^{S F B C}=A\left\{p_{2}(-\mu) E_{1}(\mu)+p_{1}(\mu) p_{1}(-\mu)\right\}
$$

\section{B. FSTD Channel Capacity Analysis}

Following the same approach for the analysis of $2 \times 1$ SFBC scheme, we determine the average channel capacity for the $(4 \times 2)$ FSTD scheme. The expression for the $k^{\text {th }}$ OFDM subcarrier $\bar{C}_{k}^{F S T D}$ is given as

$$
\bar{C}_{k}^{F S T D}=\int_{0}^{\infty} R_{C} \log _{2(1+\gamma)} f_{\gamma}^{F S T D}(\gamma) d \gamma
$$

Where $f_{\gamma}^{F S T D}(\gamma)$ is defined by:

$$
f_{\gamma}^{F S T D}(\gamma)=\frac{8}{3 \gamma^{-4}} \gamma^{3} e^{-\frac{2}{\gamma} \gamma}
$$

Substituting (25) in (24), we obtain

$$
\begin{aligned}
& \bar{C}_{k}^{F S T D}=\int_{0}^{\infty} R_{c} \log _{2}(1+\gamma) \frac{8}{3 \gamma^{-4}} \gamma^{3} e^{-\frac{2}{\gamma} \gamma} \\
& \bar{C}_{k}^{F S T D}=\frac{A \mu}{3} \int_{0}^{\infty} \ln (1+\gamma)(\mu \gamma)^{-1} e^{-\mu \gamma} d \gamma
\end{aligned}
$$

Where, $A$ and $\mu$ are as defined in (19) and $\eta=4$. Using (20), a closed form can be found as:

$$
\bar{C}_{k}^{F S T D}=2 A\left[p_{4}(-\mu) E_{1}(\mu)+\sum_{q=1}^{3} \frac{1}{q} p_{q}(\mu) p_{\eta-q}(-\mu)\right]
$$

\section{Throughput Performance With M-Qam Modulation Schemes}

In SISO OFDM systems, depends on the available bandwidth and the parameters associated with the OFDM signal, such as the number of subcarriers and the modulation order (QPSK, 16-QAM, 64-QAM). For a given frequency bandwidth $(B)$ the maximal coded data throughput in bits per second can be approximated by the following simple equation:

$$
\text { ClosedThroughput(bps) }=\frac{N_{F B} \cdot N_{S C} \cdot N_{O F D M} \cdot N_{b} \cdot E C R}{T_{s u b}}
$$

where $\mathrm{N}_{\mathrm{FB}}$ is the number of Frequency Blocks in the given frequency bandwidth $(B), \mathrm{N}_{\mathrm{SC}}$ is the number of subcarriers in one Frequency Block, $\mathrm{N}_{\text {OFDM }}$ is the number of OFDM symbols in one sub frame, $N_{b}$ is the number of bits in one subcarrier; ECR is the Effective Code Rate, and $T_{s u b}$ is the duration of one sub frame that is equal to $1 \mathrm{~ms}$. In LTE, $\mathrm{N}_{\mathrm{SC}}$ and $\mathrm{N}_{\mathrm{OFDM}}$ are fixed with values 12 and 14 respectively [4]. For $5 \mathrm{MHz}\left(\mathrm{N}_{\mathrm{FB}}=25\right)$ bandwidth LTE system with 64-QAM 
Modulation $\left(\mathrm{N}_{\mathrm{b}}=6\right)$ and $\mathrm{ECR}=0.9$, the maximal data throughput that can be supported by the system is then $22.68 \mathrm{Mbps}$. The un coded data throughput can be found by excluding the effective coderate

(ECR)

from the equation

$$
\text { Uncoded Throughput (bps) }=\frac{N_{F B} \cdot N_{S C} \cdot N_{\text {OFDM }} \cdot N_{b}}{T_{\text {sub }}}
$$

The throughput results can be compared to the system capacity $\bar{C}_{B}^{\chi}(\chi \in\{S F B C, F S T D\})$ of a flat fading channel as developed but adjusted by the inherent system losses. In this case, the system capacity is simply given as:

$$
\bar{C}_{B}^{\chi}=F \times B \times C^{-\chi}
$$

Where $F$ and $B$ are two correcting factors. The correcting factor $B$ can be calculated as:

$$
B=\frac{N_{S c} \cdot N_{O F D M} \cdot N_{F B}}{T_{\text {Sub }}}
$$

Where, $\mathrm{N}_{\mathrm{SC}}$ is the number of subcarriers in one Resource Block and is equal to $12, \mathrm{~N}_{\mathrm{OFDM}}$ represents the number of OFDM symbols in one sub frame and for normal cyclic prefix, it is equal to $14 . \mathrm{N}_{\mathrm{FB}}$ is the number of resource blocks (RB) that fit into the selected bandwidth (for example in $5 \mathrm{MHz}$ bandwidth we found 25 Resource Blocks). The values of $\mathrm{N}_{\mathrm{FB}}$ corresponding to the selected bandwidth $(B)$ are shown in Table 1.

Finally, $T_{\text {sub }}$ is the duration of one sub frame and is equal to $1 \mathrm{~ms}$. To take into account the losses due to the added cyclic prefix and reference symbols, a correction factor $F$ is introduced in system capacity and is given by:

$$
F=\frac{T_{\text {frame }}-T_{c p}}{T_{\text {frame }}} \times \frac{N_{S C} \times N_{\text {OFDM }} / 2-4}{N_{S C} \times N_{\text {OFDM }} \times 2}
$$

Where, $T_{\text {frame }}$ is the fixed duration frame and is equal to $10 \mathrm{~ms}$ TCP represents the total Cyclic Prefix time of all OFDM symbols within one frame and is given by:

$$
T_{c p}=\left(T_{c p_{1}}+6 \times T_{c p_{2}}\right) \times N_{s l o t}
$$

Where $T_{c p 1}$ is the duration of the first cyclic prefix of the first OFDM symbol in one slot and is equal to $5.2 \mu \mathrm{s} . T_{c p 2}$ is the duration of the cyclic prefix of the next six OFDM symbols in one slot as shown in Figure 1 and is equal to $4.7 \mu \mathrm{s} . N_{\text {slot }}$ is the number of slots within the duration of one frame and is equal to 20. The results of calculation of the system capacity based on Equation (31) for different bandwidth values are shown in Figure 3. 
7 OFDM Symbols

(Normal Cyclic Prefix)

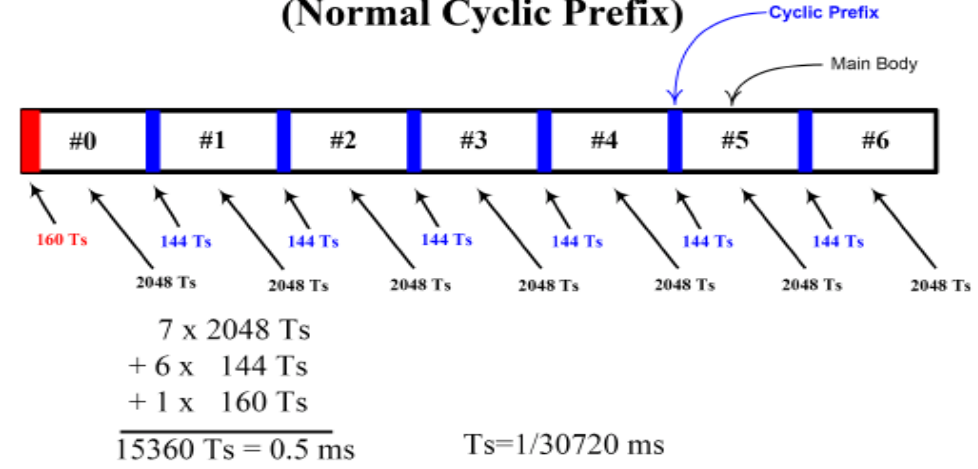

Figure 1. Structure of the symbols in one slot with Normal Cyclic Prefix.

$$
T_{c p}=\left(T_{c p_{1}}+6 \times T_{c p_{2}}\right) \times N_{s l o t}
$$

Where, Tcpl is the duration of the first cyclic prefix of the first OFDM symbol in one slot and is equal to $5.2 \mu \mathrm{s}$. Tcp2 is the duration of the cyclic prefix of the next six OFDM symbols in one slot as shown in Figure 1 and is equal to $4.7 \mu$ s. Finally, Nslot is the number of slots within the duration of one frame and is equal to 20 . The results of calculation of the system capacity based on different bandwidth values are shown in Figure 3.

The numerical results in terms of bits/s/Hz are shown in Figure 2. In 4×2 FSTD MIMO analysis, the results of Monte- Carlo simulation of channel capacity of the two diversity schemes including SISO.

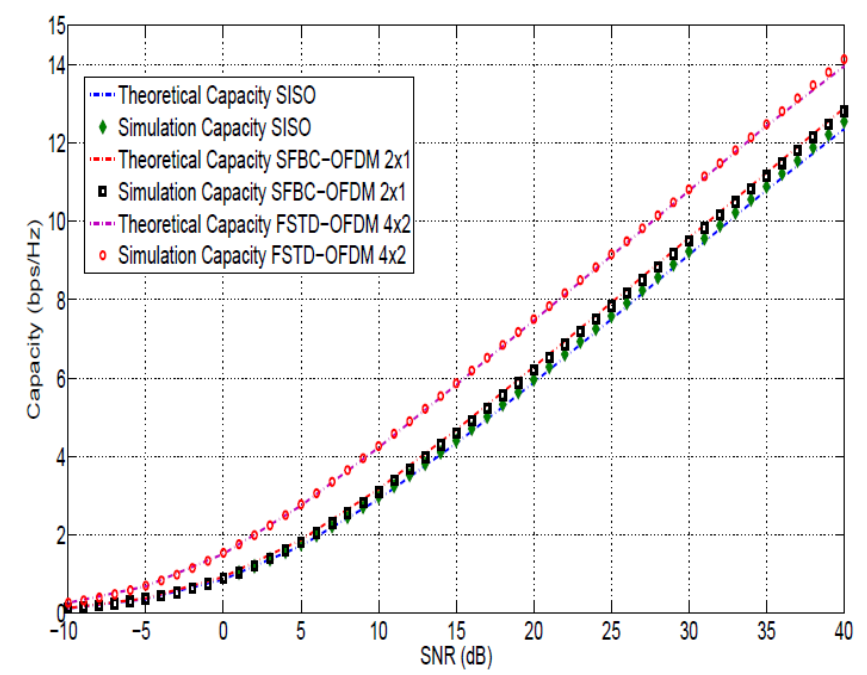

Figure 2. Capacity for SISO, SFBC-OFDM and FSTD-OFDM Schemes

The dashed line curve in Figure 2, represents the channel capacity of SISO scheme using our analysis. The diamond shaped points represents the SISO capacity obtained with Monte-Carlo 
simulation. The theoretical channel capacity of $2 \times 1$ STBC is shown by the red curve in Figure 2, whereas the black square line represent the capacity obtained by Monte-Carlo simulation. As expected, in the high SNR regions, only a small improvement of the channel capacity of the $2 \times 1$ SBC with respect to the SISO case is observed. Specifically, for SNR $=34 \mathrm{~dB}$ the improvement of $2 \times 1 \mathrm{STBC}$ is about $0.5 \mathrm{bits} / \mathrm{s} / \mathrm{Hz}$ with respect to SISO. For low SNR regions, the channel capacity of the SISO case and the $2 \times 1$ STBC is almost the same. The theoretical channel capacity of the $2 \times 2$ STBC scheme is shown by the purple dashed line in Figure 2. The improvement with respect to the SISO case is more important. For the same $\mathrm{SNR}=34 \mathrm{~dB}$, the improvement is about $1.6 \mathrm{bits} / \mathrm{s} / \mathrm{Hz}$. It can be observed from Figure 2, that numerical evaluation results obtained from capacity analysis match closely to the capacity results obtained from Monte-Carlo simulations.

The results in Figure 3, represent the limit of channel capacity of a system with continuous valued inputs and continuous valued outputs of complex signals for different channel bandwidth $(B)$ as defined in LTE (Table 1). These limits can be considered as an upper bound of real data throughput of a system with discrete valued multilevel/phase inputs. It can also be noticed from the Figure 3 how the Shannon capacity increases as the bandwidth increases.

Table 1. Number of Resource Blocks.

\begin{tabular}{|l|l|}
\hline B(MHz) & $\mathbf{N}_{\mathbf{F B}}$ \\
\hline 1.4 & 6 \\
\hline 5 & 25 \\
\hline 10 & 50 \\
\hline 15 & 75 \\
\hline 20 & 100 \\
\hline
\end{tabular}

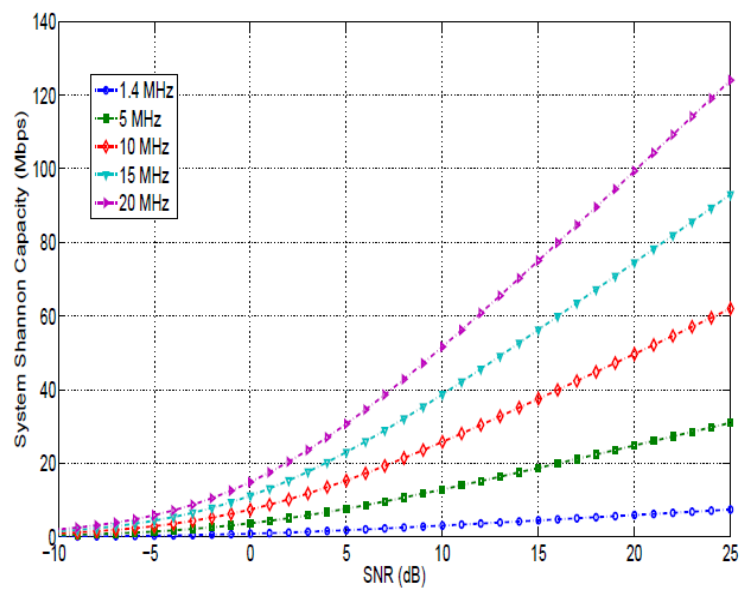

Figure 3. System Capacity as a function of SNR for different Bandwidth settings. 


\section{NUMERICAL RESULTS}

Data throughput of SFBC-OFDM and FSTD-OFDM MIMO schemes for different M-QAM modulation schemes using the LTE simulator are depicted. The LTE simulator allows the calculation of coded and un-coded data throughput. For coded calculations, the transmitted signal is coded using the Turbo Code as defined in LTE. The overall effective code rates for different modulation schemes are defined in LTE and are given in Table 2. The Monte-Carlo simulation are presented in Figure 4,5 and 6 are compared to coded and un-coded data throughput for the SISO configuration. Figure 4, represents the results for QPSK modulation. The results of 16QAM are presented in Figure 5 while those relative to 64-QAM modulation in Figure 6.

Table 2. ECR and Modulation Order for CQI.

\begin{tabular}{|l|l|l|l|}
\hline CQI & Modulation & ECR & ECR $\times \mathbf{1 0 2 4}$ \\
\hline 1 & 4QAM & 0.0762 & 78 \\
\hline 2 & 4QAM & 0.1172 & 120 \\
\hline 3 & 4QAM & 0.1885 & 193 \\
\hline 4 & 4QAM & 0.3008 & 308 \\
\hline 5 & 4QAM & 0.4385 & 449 \\
\hline 6 & 4QAM & 0.5879 & 602 \\
\hline 7 & 16QAM & 0.3691 & 378 \\
\hline 8 & 16QAM & 0.4785 & 490 \\
\hline 9 & 16QAM & 0.6016 & 616 \\
\hline 10 & 64QAM & 0.4551 & 466 \\
\hline 11 & 64QAM & 0.5537 & 567 \\
\hline 12 & 64QAM & 0.6504 & 666 \\
\hline 13 & 64QAM & 0.7539 & 772 \\
\hline 14 & 64QAM & 0.8525 & 873 \\
\hline 15 & 64QAM & 0.9258 & 948 \\
\hline
\end{tabular}

For QPSK modulation, the un-coded data throughput of SISO configuration is shown by the ball blue curve. It can be observed that as the SNR increases the data throughput increases and it reaches its maximum at an SNR of almost $48 \mathrm{~dB}$. The coded data throughput of the same modulation (QPSK) and represented by the blue curve in Figure 4 which reaches its maximum with only $10 \mathrm{~dB}$. As expected from the coding process, the coded data throughput is less than the un-coded data throughput. In fact, the coded and un-coded data throughputs are related by the effective code rate used for channel coding. As shown in Table 2, the ECR for QPSK $(C Q I=6)$ is equal to 0.5879 , which represents the ratio of the useful transmitted data rate to the overall coded data after channel coding. An ECR of 0.5879 means that only 0.5879 of the transmitted data are useful data. The remaining data are used only for error protection and detection. This principle is well represented in the results of Figure 4, where the coded data throughput is found to be 4.9 Mbps whereas the un-coded data throughput is $8.3 \mathrm{Mbps}$, which represents, effectively and as expected, a code rate of 0.5879 . The benefits of using the channel coding (Turbo coding in 
LTE)is clearly shown in Figure 4, where the data throughput of coded scheme is much closer to the channel capacity limit.

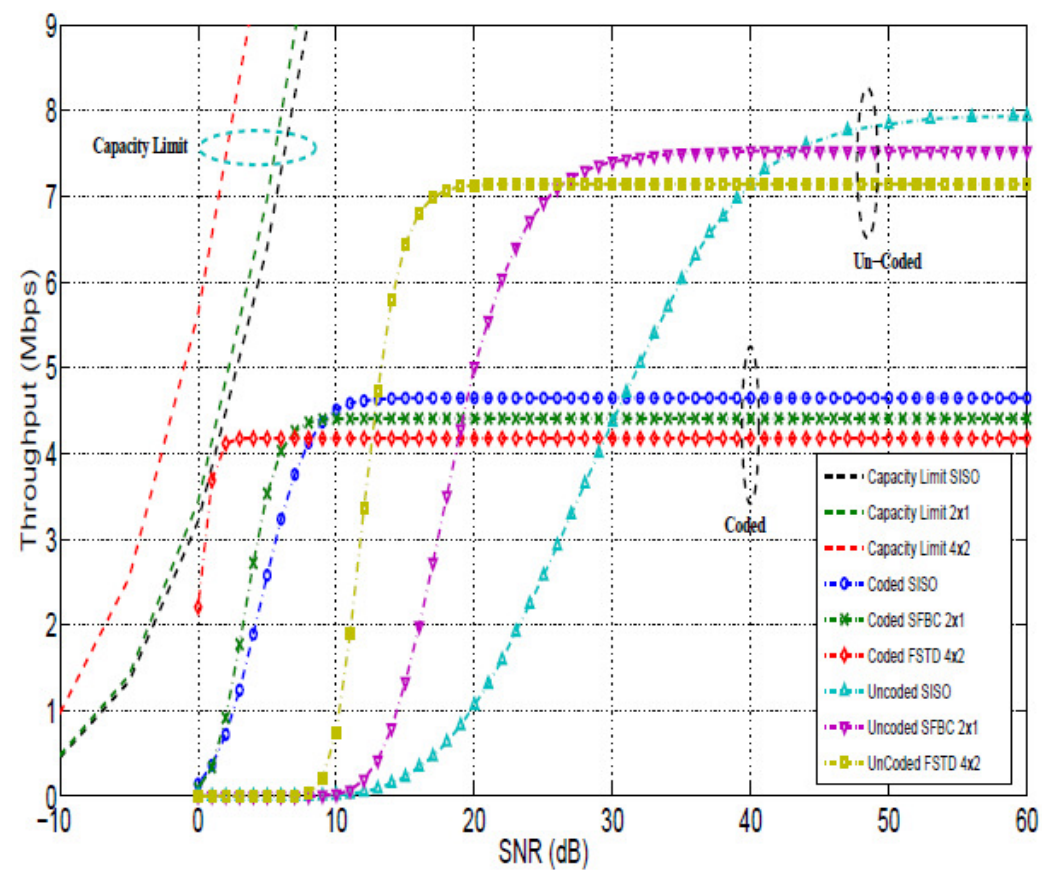

Figure 4: Data Throughput for QPSK Modulation

The coded and un-coded data throughput of the $2 \times 1$ SFBC are represented by the green and purple curves respectively. In case of un-coded scheme, the improvement with respect to the SISO case is more significant and substantial as shown in Figure 4. However, for coded case, there is no big difference between SISO and $2 \times 1$ scheme and the improvement is less significant. One last interesting observation from the results in Figure 4 is the fact that at high SNRs the SISO data throughput is greater than the data throughput for $2 \times 1$ and $4 \times 2$ diversity schemes. This result is explained by the fact that more overhead data is transmitted in case of diversity schemes as shown in Table 3. Specifically, for SISO scheme the symbol rate is 4000 per sub frame (1 ms) whereas with $2 \times 1$ SFBC case, the symbol rate is 3800 per sub frame. The symbol rate of $4 \times 2$ FSTD is the smallest one and is found to be 3600 per sub frame and that is why the data throughput of $4 \times 2$ FSTD is the smallest one at the high SNR region as shown in Figure 4. This observation is valid for both coded and un-coded data throughput. The red and citrine curves represent the coded and un-coded data throughput respectively for the $4 \times 2$ FSTD diversity scheme. Same observations as for $2 \times 1$ SFBC scheme can be made here. The theoretical channel capacity limits for SISO, $2 \times 1$ SFBC and $4 \times 2$ FSTD in a Rayleigh channel are also shown in Figure 4 for illustration and to show the effect of channel coding. It is clearly perceptible that the data throughput comes closer to the capacity limit with using channel coding. 
Table 3.4 SISO Generated Data

\begin{tabular}{|c|c|c|c|c|}
\hline \multirow{2}{*}{\multicolumn{2}{|c|}{$\begin{array}{l}\text { Modulation Scheme } \\
\text { MIMO Configuration }\end{array}$}} & \multicolumn{3}{|l|}{ QPSK } \\
\hline & & SISO & $2 \times 1$ SFBC & $4 \times 2$ FSTD \\
\hline \multicolumn{2}{|c|}{$\begin{array}{l}\text { MIMO Configuration } \\
\text { ECR }\end{array}$} & 0.5879 & 0.6016 & 0.9258 \\
\hline \multicolumn{2}{|c|}{ Max Possible Data (Bits/Sub frame) } & 8400 & 8400 & 8400 \\
\hline \multirow[b]{2}{*}{ Tx Data Bits } & $1^{\text {st }}$ Sub Frame & 4512 & 4372 & 4136 \\
\hline & $\begin{array}{l}2^{\text {nd }} \text { to } 5^{\text {th }} \text { sub } \\
\text { frame }\end{array}$ & 4680 & 4456 & 4220 \\
\hline \multirow[b]{2}{*}{ Tx Coded Bits } & $1^{\text {st }}$ Sub frame & 7712 & 7456 & 7056 \\
\hline & $\begin{array}{l}2^{\text {nd }} \text { to } 5^{\text {th }} \text { Sub } \\
\text { frame }\end{array}$ & 8000 & 7600 & 7200 \\
\hline \multirow[b]{2}{*}{ Tx User Symbols } & $1^{\text {st }}$ Sub frame & 3856 & 3728 & 3528 \\
\hline & $\begin{array}{l}2^{\text {nd }} \text { to } 5^{\text {th }} \text { Sub } \\
\text { frame }\end{array}$ & 4000 & 3800 & 3600 \\
\hline \multicolumn{2}{|c|}{$\begin{array}{l}\text { Expected Data Throughput (Bits/Sub } \\
\text { frame }\end{array}$} & 8000 & 7600 & 7200 \\
\hline \multicolumn{2}{|c|}{ Calculated ECR } & 0.5850 & 0.5863 & 0.5861 \\
\hline
\end{tabular}

For 16-QAM modulation (Figure 5), which corresponds to a CQI of 9, the code rate is 0.6016 (Table 2) and hence the un-coded data throughput is higher than the coded data throughput. For SISO scheme, the maximum un-coded and coded data throughput, represented by the ball blue and blue curves in Figure 5, are found to be $15.8 \mathrm{Mbps}$ and 9.5 Mbps respectively, which corresponds as expected to a $0.6(9.5 / 15.8)$ code rate. The maximum data throughput is achieved is achieved with an SNR of 60 scheme whereas only $20 \mathrm{~dB}$ is needed to achieve the maximum of data throughput for the coding scheme. It can be observed from Figure 5, that the best. It can be observed from Figure 5, that the best performance in terms of data throughput is achieved with the $4 \times 2$ FSTD scheme where the maximum data throughput is reached at SNR $=10 \mathrm{~dB}$. To attain the maximum in $2 \times 1$ SFBC scheme an SNR of $16 \mathrm{~dB}$ is required. For SISO scheme an SNR of $20 \mathrm{~dB}$ is needed to achieve the maximum data throughput. These results demonstrate the benefits of the diversity schemes. The code rate for CQI 15 (64-QAM Modulation) is 0.9258 which is close to 1 . The maximum un-coded and coded data throughputs are found to be 23.5 Mbps and 22 Mbps respectively, which correspond to a code rate of 0.9258 . 
International Journal of Computer Networks \& Communications (IJCNC) Vol.9, No.5, September 2017

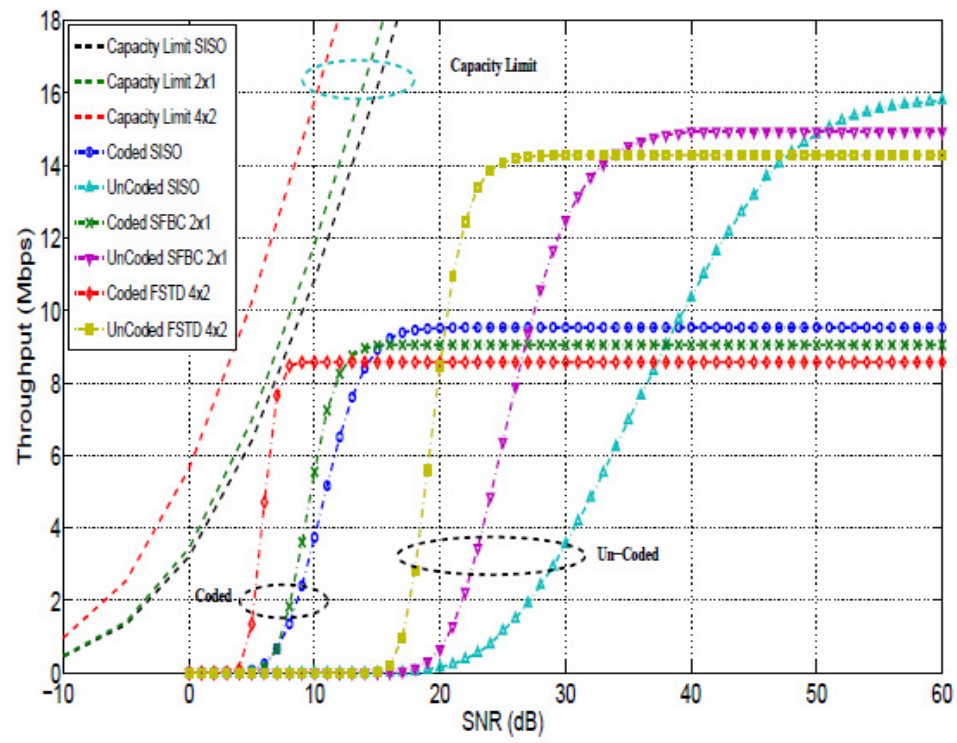

Figure 5: Data Throughput for 16QAM Modulation

The effectiveness of the FSTD scheme is clearly observed in Figure 6 where the maximum data throughput is achieved with $23 \mathrm{~dB}$ compared to $32 \mathrm{~dB}$ with $2 \times 1 \mathrm{SFBC}$ and $41 \mathrm{~dB}$ with SISO. The theoretical channel capacity limit is also shown in the Figure. To compare the coded data throughput with the theoretical limit of channel capacity, the coded data throughput of all modulation schemes and diversity schemes are made in Figure 7, where the coded data throughput for QPSK, 16-QAM, and 64-QAM and for SFBC and FSTD MIMO schemes are plotted.

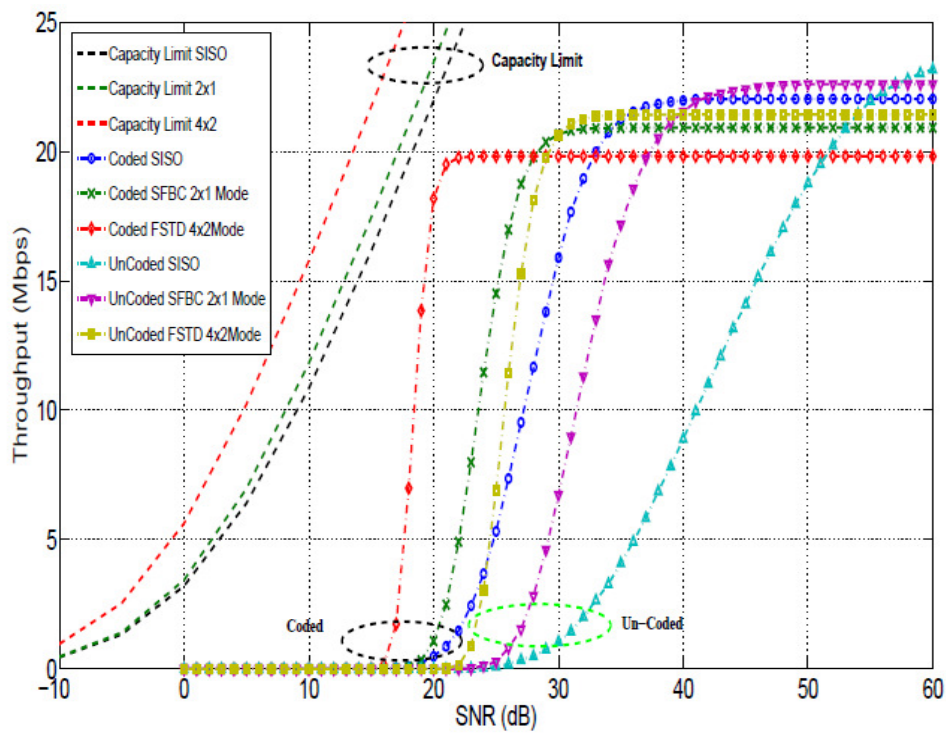

Figure 6: Data Throughput for 64QAM Modulation 


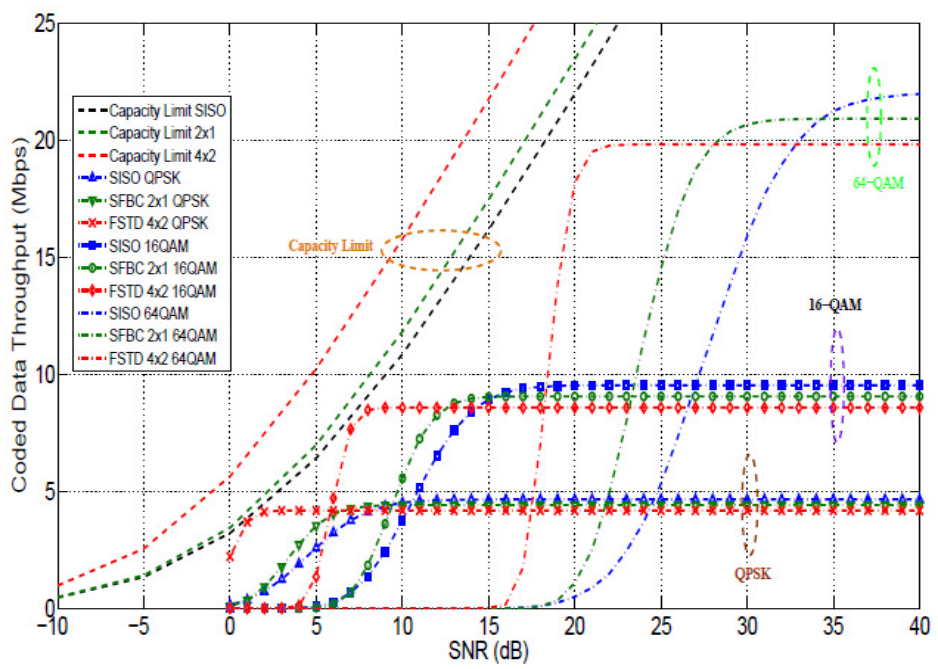

Figure 7: Coded Data Throughput for M-QAM Modulation

\section{Conclusion}

We presented an analysis and evaluation of the performance of MIMO-OFDM schemes in 4G LTE systems. More specifically, we analyzed performance for two OSTBC diversity MIMO schemes; namely the $2 \times 1$ SFBC-OFDM and the $4 \times 2$ FSTD-OFDM in the 3 GPP $5 \mathrm{MHz}$ Long Term Evolution (LTE) system over a Rayleigh flat fading channel. To show the BER performance improvement of the MIMO-OFDM schemes, the performance is compared to the SISO configuration. To ascertain the accuracy of the analysis, we also performed Monte- Carlo simulation using an LTE Link Level Simulator. The numerical results and Monte-Carlo simulation results. To complete the analysis, a study of the channel capacity for $2 \times 1$ SFBCOFDM and $4 \times 2$ FSTD-OFDM MIMO schemes in Rayleigh flat fading channel has been performed. We use the probability density function of the instantaneous signal to noise ratio to derive analytical expressions for the capacity in terms of exponential integral and Poisson distribution. Effective data throughput as measured through simulation compare well with the system channel capacity limit.

\section{REFERENCE:}

[1] 3GPP (2009). Technical specification ts36.211 version 8.7.0 "Evolved Universal Terresterial Radio Access (E-UTRA); Physical Channel and Modulation, (Release 8)".

[2] 3GPP, TR 36.913, "Requirements for further advancements for E-UTRA (LTE-Advanced)", www.3gpp.org.

[3] Progress on "LTE Advanced" - the new 4G standard Eiko Seidel, Chief Technical Officer Nomor Research GmbH, Munich, Germany.

[4] Sessia, S, Taoufik, I and Baker, M, "LTE The UMTS Long Term Evolution: From THEORY TO PRACTICE", WILEY. Second Edition, 2011.

[5] Shannon, C.E, "A mathematical theory of communication", The Bell System Technical Journal, 27, 379-423, 623-656. 
International Journal of Computer Networks \& Communications (IJCNC) Vol.9, No.5, September 2017

[6] TSE, D and Viswanath, P.TSE, D. and VISWANATH, P. "Fundamentals of Wireless Communications", Cambridge University Press, 2008.

[7] Schwarz,S., Wrulich, M. and Rupp, M., "Mutual information based calculation of the precoding matrix indicator for 3gpp UMTS/LTE Smart Antennas (WSA)" International ITG Workshop on. $52-58,2010$.

[8] Lopez-Martinez.F, Martos-Naya, E.Wong, K.K and Entrabasaguas,J, "Closed-form ber analysis of alamouti-mrc systems with icsi in ricean fading channels", Communications Letters, IEEE, 15, 46-48, 2011.

[9] Legnaian, R.Hafeez.H.M, and I.MarslandI, "BER analysis of three phase xor-and-forward relaying using Alamouti STBC". Communications Letters, IEEE, 16, 1458-1461, 2012.

[10] Gunther.c. Comment on, "Estimate of channel capacity in rayleigh fading Environment", Vehicular Technology, IEEE Transactions on, 45, 401-403, 1996.

[11] Mrs. Poornima, Prof. G. Laxminarayana and Prof. D. Srinivas Rao, "Performance of Semi-Blind Channel Estimation Approximations in LTE Downlink", Fourth International Conference on Recent Trends in Communication and Computer Networks , pp.119-128, ComNet 2016.

[12] Dr.M.Sushanth Babu and K. Ragini, "Analysis of MIMO-OFDM under Rayleigh Fading in 4G Cellular Systems", 21st International Conference on Electrical Electronics Communication Robotics and Instrumentation Engineering, (ICEECIE), pp. 1-7, 14th Aug, 2016, Mysore, INDIA.

[13] M.Sushanth Babu and Prof.K.Kishan Rao, "Fast Converging Semi-Blind SNR Estimation for Wireless MIMO-OFDM Systems," IEEE International Conference on Signal Processing, Communications and Computing. ICSPCC 2011, Xi' an, Chinna

[14] M.Sushanth Babu, Prof.K.Kishan Rao,P.Krishna, V.Ugendhar , "BER Performance Analysis for HSDPA with MIMO - SBCE Predictors", IEEE International Conference -ICICI-BME, Indonesia, Nov-2009,pp.196-200 